\title{
PHYLOGENIC, PATHOGENIC AND REPRODUCTIVE CHARACTERIZATION OF HETERORHABDITIS INDICA FROM DISTRICT MEERUT, INDIA
}

\author{
Aashaq Hussain Bhat ${ }^{1 *}$, Lovely Bharti ${ }^{2}$, Istkhar ${ }^{3}$, Aasha ${ }^{4}$ and Ashok Kumar Chaubey ${ }^{5}$ \\ $1^{*}, 2,3,4,5$ Nematology Laboratory, Department of Zoology, Chaudhary Charan Singh University, Meerut-250004.
}

India

*Corresponding Author Email: aashiqhussainbhat10@gmail.com

\section{ABSTRACT}

Heterorhabditis indica isolate $\mathrm{CH}_{18}$ was isolated from District Meerut of Uttar Pradesh, India and characterized by short juveniles and the arrangement of the terminal group of bursal ribs. The values of $E$ and $D$ ratio differentiate it from other described species of Heterorhabditis. It can be recognised by; $L=534$ (514-548) $\mu m$, pharynx $=107$ (99-111) $\mu \mathrm{m}$; ratio ' $a$ ' = 24 (22-27); ratio ' $c$ ' = 5.4 (5-6); $D \%=84$ (75-91) and $E \%=92$ (83-101). It was further recognised by the male characters viz. $L=721$ (592-884) $\mu m, S L=41$ (32-55) $\mu m ; G L=19$ (13-27) $\mu m ; G B W=46$ (33-54) $\mu \mathrm{m}$. H. indica isolate $\mathrm{CH}_{18}$ was genetically recognised by sequence lengths of the ITS region (737 bp), D2D3 region (942 bp) and COX1 (596 bp). Pathogenicity trial based on different IJ concentration viz. 25, 50, 100, 200 IJ/larva showed that the degree of susceptibility to nematode infection varied from different dose infection and also on the exposure time. A positive relation was observed in between the doses of infective juvenile and larval mortality where increments in doses also lead to increment in mortality rate. With a maximum count of progeny $\left(1.79 \pm 0.8 \times 10^{5} \mathrm{IJs} /\right.$ Larva) in lowest larval dose, a strong negative correlation was observed where the number of IJ production decreased significantly with increment in the dose of infection.

\section{KEY WORDS}

ITS region, D2-D3 region, COXI gene, pathogenicity, IJs production, negative correlation.

\section{INTRODUCTION}

Entomopathogenic nematodes, (Heterorhabditis and Steinernema) are used as potential biological control agents as they are efficient parasites of several economically important insect pests ${ }^{1,2,3}$ being symbiotically associated with entomopathogenic bacteria Photorhabdus ${ }^{4}$ and Xenorhabdus ${ }^{5}$. To date, twenty five species have been described for Heterorhabditis and more than hundred for Steinernema. There has been considerable debate about the proper identification of entomopathogenic nematodes ${ }^{6,3,7}$. Taxonomic relationships of these nematodes are usually based on morphological characters for Heterorhabditis species while for Steinemema species morphological characters are combined with cross breeding data ${ }^{8,9,7}$. Morphological characters cannot be used unambiguously to place new isolates into a particular species. The feasibility of using these nematodes as biological control agents depends upon the resources required for a rapid and accurate means to determine the genetic diversity among existing populations of entomopathogenic nematode species. These methods can also be used for the development of identification tools. The use of molecular approaches to study systematic relationships among entomopathogenic nematodes have been emphasized ${ }^{10}$. Currently regions of taxonomic importance include the internal transcribed spacer (ITS) and D2D3 region of the ribosomal DNA (rDNA). Both of these regions have been widely used for the identification of few nematodes ${ }^{11,12}$ and will make major contributions to the identification of entomopathogenic nematode species.

During a survey of sampling in cultivars of Meerut region, an isolate of Heterorhabditid nematode tagged as $\mathrm{CH}_{18}$ was isolated and based on morphological and molecular characteristics was 
identified as Heterorhabdtis indica. In the present investigation, three regions viz. ITS, D2D3 and COX1 were used to delimit its species status in addition morpho-taxometrical investigation. The pathogenicity and reproductive prospective of this isolate was also observed in the present study.

\section{MATERIALS AND METHODS}

\section{Nematode collection}

The infective juvenile (IJ) stages of EPN mostly reside in the moist soils and have been reported in almost all type of soils except dry soils. Therefore, to get hold of these pristine indigenous, local EPN, soils were collected randomly from different agricultural fields of District Meerut using appropriate sampling methods. Recovery of EPN from soil samples was scrutinized using insect baiting with the last instars of Galleria mellonella (L.) (Lepidoptera:Pyralidae) ${ }^{13}$. Maintenance of IJ were done by recycling through $G$. mellonella larvae and stored in approximately $150 \mathrm{ml}$ of sterilized distilled water in $500 \mathrm{ml}$ tissue culture flasks at $15^{\circ} \mathrm{C}$ for subsequent identification and establishment of stock cultures.

\section{Morphological Observations}

To study the morphology and morphometry, adult stages and 3rd stage juveniles were recovered from the cadaver of $G$. mellonella. For recovery of adults, the cadaver of $G$. mellonella were dissected 2-3 days and 4-6 days after mortality for hermaphroditic females and subsequent generation of amphimictic males and females respectively in Ringer's Solution ${ }^{14}$ while freshly emerged IJ were obtained from white trap $^{15}$ for the identification purpose. These all stages were killed with lukewarm water, fixed in $\mathrm{TAF}^{16}$ and subsequently processed to glycerine by the Seinhorst method $^{17}$. Nematodes were transferred to the fresh drop of glycerine on a microscope slide, crystals of paraffin wax placed around the glycerin drop and covered with a cover glass and placed on hot plate for melting of wax for sealing.

Morphometric analysis of the nematode specimens was done for 15 individuals of the adult stages of generations and infective juveniles (IJ), using light microscopy and the image analyzing software DL-S1 (Phase contrast, Japan).

\section{Molecular Characterization and Phylogenetic Relationships}

Genomic DNA was extracted from 3rd stage infective juveniles through Quigen DNA Blood and Tissue Kit as per manufacturer's instruction with slight modifications. Agarose Gel Electrophoresis (AGE) was performed to detect the presence of DNA in the eluted solution and the primers suggested by Joyce et al. $^{18}$ were used for PCR. The amplification of the ribosomal and mitochondrial regions was carried out using Dream Taq Green PCR Master Mix 2X (Thermo Scientific) in $100 \mu$ leppendorf (AB Scientific) with a final reaction volume of $30 \mu \mathrm{l}$. Amplifications were carried out using Verti 96 well fast thermocycler with heated lid pre-set at $95{ }^{\circ} \mathrm{C}$ and subjected to the following cycling profile: For the ITS rDNA region, PCR conditions included initial denaturation at $94{ }^{\circ} \mathrm{C}$ for 5 min, followed by 33 cycles of $94{ }^{0} \mathrm{C}$ for $1 \mathrm{~min}, 55{ }^{\circ} \mathrm{C}$ for $1 \mathrm{~min} 30 \mathrm{sec}$ and $72{ }^{\circ} \mathrm{C}$ for $2 \mathrm{~min}$, followed by a final extension at $72{ }^{\circ} \mathrm{C}$ for $5 \mathrm{~min}$ to ensure all of the amplification products are full length.. For the $28 \mathrm{~S}$ (D2D3) rDNA region, the parameters included denaturation at 94 o $\mathrm{C}$ for $5 \mathrm{~min}$, followed by 33 cycles of $94{ }^{\circ} \mathrm{C}$ for $30 \mathrm{sec}, 52{ }^{\circ} \mathrm{C}$ for $30 \mathrm{sec}$ and $72{ }^{\circ} \mathrm{C}$ for 1 $\mathrm{min}$, followed by a final extension at 72 o $\mathrm{C}$ for $7 \mathrm{~min}$. For the COX1 mtDNA region, the PCR protocol included denaturation at $94{ }^{\circ} \mathrm{C}$ for $3 \mathrm{~min}$, followed by 37 cycles of $94{ }^{\circ} \mathrm{C}$ for $30 \mathrm{sec}, 50{ }^{\circ} \mathrm{C}$ for $30 \mathrm{sec}$ and $72{ }^{\circ} \mathrm{C}$ for $45 \mathrm{sec}$, followed by a final extension at $72{ }^{\circ} \mathrm{C}$ for 7 min. Electrophoresis of the PCR products was carried in a $1 \%$ TAE buffered agarose gel stained with $\mathrm{EtBr}$. The products were analyzed on $1 \%$ agarose gel with TAE buffer.

The amplified PCR products were purified and sequenced in both directions by Bioserve Biotechnologies Pvt. Ltd, Hyderabad, India. The contig sequences were deposited in NCBI GenBank database under the following accession numbers; ITS regions (KU176726), D2-D3 regions (KU176127) of rDNA and COX1 (KU306237) for mtDNA. The sequences were edited and compared with those present in the Genbank by means of a Basic Local Alignment Search Tool (BLAST). An alignment of samples together with sequences of the closely related species of the Heterorhabditis was produced for each amplified DNA region using default Crustal $W$ parameters in MEGA 
6.0 (Tamura et al., 2013) and optimized manually in BioEdit. Pairwise distances and phylogenetic trees (Maximum Parsimony) were computed using MEGA $6.0^{19}$. Codon positions included were first + second + third + non-coding.

Bioassay experimentation for Pathogenicity and Reproductive Potential

Pathogenicity and reproductive potential of the present species was carried out at different inoculums viz. 25, 50, 100 and $200 \mathrm{IJ} /$ Larvae. For evaluating pathogenicity, freshly emerged IJ were used for larval mortality against $G$. mellonella in 6 well plates $(3.5 \mathrm{~cm}$ diameter) lined by double Whatman filter paper No. 1. Four different concentrations viz. 25, 50, 100 and 200 IJ were prepared separately in DDW. The total moisture with each IJ dose was maintained $450 \mu \mathrm{l}$ with distilled water and was poured into each well with the help of micro pipette in the well plates separately. IJ viability was confirmed under stereo microscope (NIKON SMZ 645) before pouring into wells. Larvae of same size and weight were infected in 6 well plates and all experiments were replicated 10 times along with control for each treatment and incubated at $28 \pm 2{ }^{\circ} \mathrm{C}$ in BOD till the mortality of larvae. After the death, all the larvae were transferred onto the white trap for the emergence of IJ to confirm that the mortality was due to IJ infection. Mortality was recorded after each $12 \mathrm{~h}$ interval till $100 \%$ mortality was achieved and the same cadavers were utilized to compute the progeny production of IJ. The IJ started to emerge from the cadavers into the white trap after 5-7 days of transfer and the emerged IJ from each larva were collected till their emergence (18-20 days) stopped and were transferred from production dish to a culture flask separately in DDW. The amount of DDW was maintained to $50 \mathrm{ml}$ and the nematodes were counted under stereomicroscope (NIKON SMZ 645 ) in $1 \mathrm{ml}$ suspension with the help of counting dish. The total progeny production was determined with the help of statistical tools.

\section{Statistical Analysis}

Data obtained through measurements of De Man Indices was analyzed statistically where the descriptive analysis was performed and data was presented in measurement in $\mu \mathrm{m} \pm \mathrm{SD}$ (range) except the ratios and percentage values. The insect larval mortality assay was analyzed statistically through probit analysis and $\mathrm{LC}_{50}$ and $\mathrm{LT}_{50}$ values were calculated at $95 \%$ confidence limit. All the mortality recorded in the form of percentage mortality and graphical presentations were made using excel.

Total No. of IJ/Larva of the studied nematode was analyzed by descriptive analysis and presented in number of IJ \pm SE (range). Analysis of variance (One way ANOVA) was performed to find out the significance relationship between IJ penetration and production with a significance level of $\mathrm{P}<0.05$.

\section{RESULTS AND DISCUSSION}

\section{Morphological Observations}

The $\mathrm{H}$. indica isolate $\mathrm{CH}_{18}$ was isolated from the soils of sugarcane fields (Saccharum officinarum L.) of Baksar area of district Meerut, Uttar Pradesh, India. Present isolate was characterized by the short juveniles almost similar to the original description and the varied arrangement of the terminal group of bursal ribs. The values of $E$ and $D$ ratio differentiate it from other described species of Heterorhabditis. It can be recognised by IJ body diam. = $22(20-24) \mu \mathrm{m}, \mathrm{L}=$ 534 (514-548) $\mu \mathrm{m}$, pharynx $=107(99-111) \mu \mathrm{m}$; ratio ' $a$ ' = 24 (22-27); ratio ' $c$ ' = 5.4 (5-6); D\% = 84 (75-91) and $E \%=92$ (83-101). It was further recognised by the male characters viz. $\mathrm{L}=721(592-884) \mu \mathrm{m}, \mathrm{SL}=41$ (32-55) $\mu \mathrm{m} ; \mathrm{GL}=19$ (13-27) $\mu \mathrm{m} ; \mathrm{GBW}=46$ (33-54) $\mu \mathrm{m}$.

$H$. indica isolate $\mathrm{CH}_{18}$ was compared with six closely related species of Heterorhabditis, and it differed in some morphological and morphometric characters with compared species but showed much similitude with $\mathrm{H}$. indica. The comparison of the morphometrics with other species is presented in the Tables (Table 1 \& 2) and is described as follows:

$\mathrm{H}$. indica isolate $\mathrm{CH}_{18}$ was separated from $\mathrm{H}$. taysearae by longer body of IJ 534 (514-548) vs. 418 (332-499) $\mu \mathrm{m}$; ratio ' $a$ ' =24 (22-27) vs. 21 (18-27); ratio ' $b$ ' = 5 (4.74-5.47) vs. $3.8(3.4-4.2)$ and shorter ratio ' $c$ ' = 5.46 (4.97-5.95) vs. 7 (6.5-8.7) $\mu \mathrm{m} ; \mathrm{E} \%=92$ (83-101) vs. 180 (110-230) and tail 98 (89-106) vs. 55 (44-70) $\mu \mathrm{m}$. Males of the present specimen can be differentiated from $H$. taysearae by $\mathrm{SL}=41(32-55)$ vs. 39 (30-42) $\mu \mathrm{m} ; \mathrm{GBW}=46$ (33 -54) vs. 43 (38-48) $\mu \mathrm{m} ; \mathrm{EP}=74$ (65$86)$ vs. 95 (78-120) $\mu \mathrm{m} ; \mathrm{ES}=88$ (78-96) vs. 112 (85- 
123) $\mu \mathrm{m} ; \mathrm{TR}=101$ (75-131) vs. $122(100-146) \mu \mathrm{m}$ and $\quad$ SW\% $=200$ (113-299) vs. 156

Table 1. Comparative morphometrics of infective juveniles of $\boldsymbol{H}$. indica $\mathrm{CH}_{18}$ and related Heterorhabditis spp. (in ascending order of body length). Measurements are in $\mu \mathrm{m}$ (expect $n$, ratio and percentage) and in the form: mean (range). Data for $\mathrm{H}$. indica $\mathrm{CH}_{18}$ in bold.

\begin{tabular}{|c|c|c|c|c|c|c|c|}
\hline Characters & taysearae & indica & $\mathrm{CH}_{18}$ & baujardi & floridensis & mexicana & bacteriophora \\
\hline $\mathbf{n}$ & 30 & 25 & 15 & 25 & 25 & 25 & 15 \\
\hline I & $\begin{array}{c}418 \\
(332-499)\end{array}$ & $\begin{array}{c}528 \\
(479-573)\end{array}$ & $\begin{array}{c}534 \\
(514-548)\end{array}$ & $\begin{array}{c}551 \\
(497-595)\end{array}$ & $\begin{array}{c}562 \\
(554-609)\end{array}$ & $\begin{array}{c}578 \\
(530-620)\end{array}$ & $\begin{array}{c}588 \\
(512-617)\end{array}$ \\
\hline a & $\begin{array}{c}21 \\
(18-27) \\
\end{array}$ & $\begin{array}{c}26 \\
(25-27) \\
\end{array}$ & $\begin{array}{c}24 \\
(22-27) \\
\end{array}$ & $\begin{array}{c}28 \\
(26-30) \\
\end{array}$ & $\begin{array}{c}27 \\
(25-32) \\
\end{array}$ & $\begin{array}{c}25 \\
(23.6-28.4) \\
\end{array}$ & $\begin{array}{c}25 \\
(17-30) \\
\end{array}$ \\
\hline b & $\begin{array}{c}3.8 \\
(3.4-4.2) \\
\end{array}$ & $\begin{array}{c}4.5 \\
(4.3-4.8) \\
\end{array}$ & $\begin{array}{c}5 \\
(4.7-5.5) \\
\end{array}$ & $\begin{array}{c}4.8 \\
(4.5-5.1) \\
\end{array}$ & $\begin{array}{c}4.3 \\
(3.9-4.9) \\
\end{array}$ & $\begin{array}{c}4.6 \\
(4.2-5.1) \\
\end{array}$ & $\begin{array}{c}4.5 \\
(4.0-5.1) \\
\end{array}$ \\
\hline C & $\begin{array}{c}7.7 \\
(6.5-8.7) \\
\end{array}$ & $\begin{array}{c}5.3 \\
(4.5-5.6) \\
\end{array}$ & $\begin{array}{c}5.4 \\
(5-6) \\
\end{array}$ & $\begin{array}{c}6 \\
(6-6.7) \\
\end{array}$ & $\begin{array}{c}5.6 \\
(5.3-6.6) \\
\end{array}$ & $\begin{array}{c}5.9 \\
(5.5-6.3) \\
\end{array}$ & $\begin{array}{c}6.2 \\
(5.5-7.0) \\
\end{array}$ \\
\hline GBW & $\begin{array}{c}20 \\
(17-23) \\
\end{array}$ & $\begin{array}{c}20 \\
(19-23) \\
\end{array}$ & $\begin{array}{c}22 \\
(20-24) \\
\end{array}$ & $\begin{array}{c}20 \\
(18-22) \\
\end{array}$ & $\begin{array}{c}21 \\
(19-23) \\
\end{array}$ & $\begin{array}{c}23 \\
(20-24) \\
\end{array}$ & $\begin{array}{c}23 \\
(18-31) \\
\end{array}$ \\
\hline EP & $\begin{array}{c}90 \\
(74-113)\end{array}$ & $\begin{array}{c}98 \\
(88-107)\end{array}$ & $\begin{array}{c}90 \\
(82-95)\end{array}$ & $\begin{array}{c}97 \\
(91-103)\end{array}$ & $\begin{array}{c}109 \\
(101-122) \\
\end{array}$ & $\begin{array}{c}102 \\
(83-109)\end{array}$ & $\begin{array}{c}103 \\
(87-110)\end{array}$ \\
\hline NR & $\begin{array}{c}64 \\
(58-87) \\
\end{array}$ & $\begin{array}{c}82 \\
(72-85) \\
\end{array}$ & $\begin{array}{c}78 \\
(70-85) \\
\end{array}$ & $\begin{array}{c}81 \\
(75-86) \\
\end{array}$ & $\begin{array}{c}86 \\
(68-107) \\
\end{array}$ & $\begin{array}{c}81 \\
(74-88) \\
\end{array}$ & $\begin{array}{c}85 \\
(72-93) \\
\end{array}$ \\
\hline ES & $\begin{array}{c}110 \\
(96-130)\end{array}$ & $\begin{array}{c}117 \\
(109-123) \\
\end{array}$ & $\begin{array}{c}107 \\
(99-111)\end{array}$ & $\begin{array}{c}115 \\
(107-120) \\
\end{array}$ & $\begin{array}{c}135 \\
(123-142) \\
\end{array}$ & $\begin{array}{c}122 \\
(104-142) \\
\end{array}$ & $\begin{array}{c}125 \\
(100-139) \\
\end{array}$ \\
\hline Tail with sheath & $\begin{array}{c}55 \\
(44-70) \\
\end{array}$ & $\begin{array}{c}101 \\
(93-109) \\
\end{array}$ & $\begin{array}{c}98 \\
(89-106) \\
\end{array}$ & $\begin{array}{c}90 \\
(83-97) \\
\end{array}$ & $\begin{array}{c}103 \\
(91-113) \\
\end{array}$ & $\begin{array}{c}99 \\
(91-106) \\
\end{array}$ & $\begin{array}{c}98 \\
(83-112) \\
\end{array}$ \\
\hline ABW & - & - & $\begin{array}{c}15 \\
(13-17)\end{array}$ & $\begin{array}{c}13 \\
(11-14)\end{array}$ & $14(12-16)$ & $\begin{array}{c}15 \\
(12-17)\end{array}$ & - \\
\hline D\% & $\begin{array}{c}82 \\
(71-96) \\
\end{array}$ & $\begin{array}{c}84 \\
(79-90) \\
\end{array}$ & $\begin{array}{c}84 \\
(75-91)\end{array}$ & $\begin{array}{c}84 \\
(78-88) \\
\end{array}$ & $\begin{array}{c}81 \\
(71-90)\end{array}$ & $\begin{array}{c}81 \\
(72-86) \\
\end{array}$ & $\begin{array}{c}84 \\
(76-92) \\
\end{array}$ \\
\hline E\% & $\begin{array}{c}180 \\
(110-230)\end{array}$ & $\begin{array}{c}94 \\
(83-103)\end{array}$ & $\begin{array}{c}92 \\
(83-101)\end{array}$ & $\begin{array}{c}108 \\
(98-114)\end{array}$ & $\begin{array}{c}105 \\
(95-134)\end{array}$ & $\begin{array}{c}104 \\
(87-111)\end{array}$ & $\begin{array}{c}112 \\
(103-130)\end{array}$ \\
\hline
\end{tabular}


Table 2. Comparative morphometrics of first-generation males of $\boldsymbol{H}$. indica $\mathrm{CH}_{18}$ and related Heterorhabditis spp. Measurements are in $\mu \mathrm{m}$ (expect $\mathrm{n}$, ratio and percentage) and in the form: mean (range). Data for $\boldsymbol{H}$. indica $\mathrm{CH}_{18}$ in bold.

\begin{tabular}{|c|c|c|c|c|c|c|c|}
\hline Characters & taysearae & Indica & $\mathrm{CH}_{18}$ & baujardi & floridensis & mexicana & bacteriophora \\
\hline $\mathbf{N}$ & 20 & 12 & 15 & 14 & 20 & 20 & 15 \\
\hline \multirow{2}{*}{$\mathbf{L}$} & 703 & 721 & 721 & 889 & 862 & 686 & 820 \\
\hline & (648-736) & $(573-788)$ & (592-884) & (818-970) & (785-924) & $(614-801)$ & $(780-960)$ \\
\hline \multirow{2}{*}{ GBW } & 43 & 42 & 46 & 49 & 47 & 42 & 43 \\
\hline & $(38-48)$ & $(35-46)$ & $(33-54)$ & $(45-53)$ & $(43-50)$ & $(38-47)$ & $(38-46)$ \\
\hline \multirow{2}{*}{ EP } & 95 & 123 & 74 & 81 & 117 & 124 & 121 \\
\hline & $(78-120)$ & $(109-138)$ & $(65-86)$ & $(71-93)$ & $(104-128)$ & $(108-145)$ & $(114-130)$ \\
\hline \multirow{2}{*}{ NR } & 65 & 75 & 65 & 65 & 80 & 71 & 72 \\
\hline & $(54-88)$ & $(72-85)$ & $(56-74)$ & (54-77) & $(73-90)$ & $(61-83)$ & $(65-81)$ \\
\hline \multirow{2}{*}{ ES } & 112 & 101 & 89 & 116 & 105 & 96 & 103 \\
\hline & $(85-1230$ & (93-109) & $(78-96)$ & $(105-132)$ & $(97-111)$ & (89-108) & (99-105) \\
\hline \multirow{2}{*}{ TR } & 122 & 91 & 101 & 91 & 93 & 96 & 79 \\
\hline & $(100-146)$ & $(35-144)$ & $(75-131)$ & $(28-38)$ & $(78-116)$ & $(65-130)$ & $(59-87)$ \\
\hline \multirow{2}{*}{ SL } & 39 & 43 & 41 & 40 & 42 & 41 & 40 \\
\hline & $(30-42)$ & $(35-48)$ & (32S-55) & $(33-45)$ & $(36-46)$ & $(30-47)$ & $(36-44)$ \\
\hline \multirow{2}{*}{ GL } & 18 & 21 & 19 & 20 & 23 & 23 & 20 \\
\hline & $(14-21)$ & $(18-23)$ & $(13-27)$ & $(18-22)$ & $(17-30)$ & $(18-32)$ & $(18-25)$ \\
\hline \multirow{2}{*}{ D\% } & \multirow{2}{*}{88} & \multirow{2}{*}{121} & 84 & & 112 & 129 & \multirow{2}{*}{117} \\
\hline & & & $(68-93)$ & - & $(105-119)$ & $(114-149)$ & \\
\hline \multirow{2}{*}{ sW } & \multirow{2}{*}{156} & \multirow{2}{*}{187} & 200 & 182 & 157 & 167 & \multirow{2}{*}{174} \\
\hline & & & (113-299) & $(138-208)$ & $(133-209)$ & $(130-196)$ & \\
\hline \multirow{2}{*}{ GS } & \multirow{2}{*}{46} & \multirow{2}{*}{49} & 46 & 50 & 53 & 56 & \multirow{2}{*}{50} \\
\hline & & & $(38-56)$ & (44-61) & $(47-65)$ & $(43-70)$ & \\
\hline
\end{tabular}

-, Data not available.

Morphologically $H$. indica $\mathrm{CH}_{18}$ was separated from $H$. baujardi by the shape of gubernaculum proximal end bearing knob vs. lacking, ventrally straight vs. curved. Males of $H$. indica $\mathrm{CH}_{18}$ differs from $H$. baujardi by $\mathrm{ES}=$ 89 (78-95) vs. $116(105-132) \mu \mathrm{m}, \mathrm{TR}=101$ (75-131) vs. $91(28-38) \mu \mathrm{m}$, Tail $=27(18-42)$ vs. $33(28-38) \mu \mathrm{m}$, SW\% $=200(113-299)$ vs. $182(138-208)$ and GS\% = 46 (38-56) vs. 50 (44-61). The IJ of the present isolate differs from $H$. baujardi by having shorter body length 534 (514-548) vs. 551 (497-595) $\mu \mathrm{m}$, ratio 'a' = 24 (2227) vs. 28 (26-30), EP = 90 (82-95) vs. 97 (91-103) $\mu \mathrm{m}$, $E \%=92(83-101)$ vs. 108 (98-114) and tail 98 (89-106) vs. 90 (83-97) $\mu \mathrm{m}$.

$H$. indica $\mathrm{CH}_{18}$ males were differentiated from $H$. floridensis males by a EP $=74$ (65-86) vs. 117 (104128) $\mu \mathrm{m}, \mathrm{NR}=65$ (56-74) vs. $80(73-90) \mu \mathrm{m}, \quad E S=89$ (78-96) vs. 105 (97-111) $\mu \mathrm{m}, \mathrm{GL}=19$ (13-27) vs. 23
(17-30) $\mu \mathrm{m}, \mathrm{SW} \%=200$ (113-299) vs. 157 (133-209), GS\%. $46(38-56)$ vs. $54(47-65)$ and D\% = 84 (68-93) vs. 112 (105-119). IJ were shorter than $H$. floridensis 534 (514-548) vs. 562 (554-609) $\mu \mathrm{m}$, Tail = 98 (89-106) vs. $103(91-113) \mu \mathrm{m}$ and ratio 'a' = 24 (22 -27) vs. 27 (25-32), E\% = 92 (83-101) vs. 101 (95-134).

Male of $H$. indica $\mathrm{CH}_{18}$ was separated from $H$. mexicana by $\mathrm{GBW}=46$ (33-53) vs. $42(38-47) \mu \mathrm{m}, \mathrm{EP}=$ $74(65-86)$ vs. $124(108-145) \mu \mathrm{m}, \mathrm{GL}=19$ (13-27) vs. $23(18-32) \mu \mathrm{m}, \mathrm{D} \%=83$ (68-93) vs. 129 (114-149), $S W \%=200$ (113-299) vs. 167 (133-196), GS\%.= 46 (3856) vs. 56 (43-70). IJ can be distinguished by the body length $=534$ (514-548) vs. 578 (530-620) $\mu \mathrm{m}, \mathrm{EP}=90$ (81-94) vs. 102 (83-109) $\mu \mathrm{m}, \mathrm{ES}=107$ (99-111) vs. 122 $(104-142) \mu \mathrm{m}, \mathrm{D} \%=84$ (75-91) vs. 81 (72-86) and $\mathrm{E} \%=$ 92 (83-101) vs. 104 (87-111). The vulval pattern in 
females is different in both specimens which also separated them from one another.

Males of $H$. indica $\mathrm{CH}_{18}$ was differentiated from $\mathrm{H}$. bacteriophora males by SL $=41$ (32-55) vs. 40 (36-44) $\mu \mathrm{m}, \mathrm{GL}=19$ (13-27) vs. $20(18-25) \mu \mathrm{m}, \mathrm{D} \%=84$ (68$93)$ vs. $117, A B W=21$ (17-29) vs. $23(22-25) \mu \mathrm{m}, \mathrm{sW} \%$ $=200$ (113-299) vs. 174 and GS\% = 46 (38-56) vs. 50 . IJ can be characterised by the shorter body length 534 (514-548) vs. 588 (512-617) $\mu \mathrm{m}, \mathrm{NR}=78$ (70-85) vs. 85 (72-93) $\mu \mathrm{m}, \mathrm{EP}=90$ (81-94) vs. $103(87-110) \mu \mathrm{m}$, ratio 'a' = 24 (21-26) vs. 25 (17-30) $\mu \mathrm{m}$, ES = 107 (99-111) vs. 125 (100-139) $\mu \mathrm{m}$ and $E \%=92$ (83-101) vs. 112 (103-130).

Molecular Characterization and Phylogenetic Relationships

$H$. indica isolate $\mathrm{CH}_{18}$ was characterised genetically by sequences of ITS (KU176726), D2-D3 regions (KU176127) of rDNA and COX1 (KU306237) mt DNA.
The quality of PCR products of said genes was confirmed by agarose gel electrophoresis. The length of the internal transcribed spacer region was $737 \mathrm{bp}$ with ITS1 $=369 \mathrm{bp}, 5.8 \mathrm{~S}=154 \mathrm{bp}$ and ITS2 $=214 \mathrm{bp}$ and its nucleotide composition was: $\mathrm{A}=26.46 \%, \mathrm{C}=$ $19.95 \%, G=24.83 \%, T=28.77 \%$. The length of the total sequence is the smallest among the 14 related species of Heterorhabditis (Table 3 ). $\mathrm{H}$. indica isolate $\mathrm{CH}_{18}$ differed from $\mathrm{H}$. baujardi, $\mathrm{H}$. bacteriophora, $\mathrm{H}$. floridensis and $H$. mexicana from its closest taxon by 25 bp, 34 bp, 23 bp and 24 bp, respectively while with already described $H$. indica (AY321483) by only 2 bp and thus not much variation, hence considered same. Pairwise distance of ITS and D2D3 regions of rDNA are shown in Table 4-5. Both the regions of taxonomic importance showed $100 \%$ percentage similarity with the original specimen.

Table 3. Sequence lengths and nucleotide composition of ITS (ITS1 + 5.8S + ITS2) and D2-D3 regions of species of Heterorhabditis closely related to $\mathrm{H}$. indica $\mathrm{CH}_{18}$. Data of $\mathrm{H}$. indica $\mathrm{CH}_{18}$ in bold.

\begin{tabular}{|c|c|c|c|c|c|c|c|c|c|c|}
\hline \multirow{2}{*}{$\begin{array}{l}\text { Species } \\
\text { ITS }\end{array}$} & \multirow[b]{2}{*}{ Acc. No. } & \multicolumn{7}{|c|}{ Molecular markers } & \multirow{2}{*}{ ITS(bp) } & \multirow{2}{*}{ Seq. (bp) } \\
\hline & & ITS1(bp) & $5.8 S(b p)$ & ITS2(bp) & A & C & G & $T$ & & \\
\hline H. indica $\mathrm{CH}_{18}$ & KU176126 & 369 & 154 & 214 & 26.46 & 19.95 & 24.83 & 28.77 & 739 & \\
\hline H. indica & AY321483 & 370 & 154 & 215 & 20.78 & 21.43 & 28.57 & 29.22 & 739 & \\
\hline H. noenieputensis & JN620538 & 371 & 154 & 216 & 20.78 & 21.43 & 28.57 & 29.22 & 741 & \\
\hline H. amazonens & DQ665222 & 395 & 154 & 211 & 21.43 & 21.43 & 28.57 & 28.57 & 760 & \\
\hline H. baujardi & AF548768 & 397 & 153 & 212 & 20.92 & 21.57 & 28.76 & 28.76 & 762 & \\
\hline H. floridens & DQ372922 & 393 & 154 & 213 & 21.43 & 21.43 & 28.57 & 28.57 & 760 & \\
\hline H. Mexicana & AY321478 & 394 & 154 & 213 & 22.08 & 21.43 & 28.57 & 27.92 & 761 & \\
\hline H. bacteriophora & AY321477 & 389 & 154 & 228 & 21.43 & 20.78 & 28.57 & 29.22 & 771 & \\
\hline H. Georgiana & EU099032 & 389 & 154 & 228 & 22.08 & 20.13 & 28.57 & 29.22 & 771 & \\
\hline H. atacamensis & HM230723 & 350 & 154 & 211 & 20.78 & 21.43 & 29.22 & 28.57 & 715 & \\
\hline H. safricana & EF488006 & 379 & 154 & 211 & 22.08 & 19.48 & 29.22 & 29.22 & 744 & \\
\hline H. marelatus & AY321479 & 379 & 154 & 211 & 21.43 & 21.43 & 28.57 & 28.57 & 744 & \\
\hline H. zealandica & AY321481 & 387 & 154 & 212 & 21.43 & 22.08 & 27.92 & 28.57 & 753 & \\
\hline H. downesi & AY321482 & 374 & 154 & 212 & 21.43 & 21.43 & 28.57 & 28.57 & 740 & \\
\hline H. megidis & AY321480 & 384 & 154 & 220 & 22.08 & 21.43 & 28.57 & 27.92 & 758 & \\
\hline \multicolumn{11}{|l|}{ D2D3 } \\
\hline H. indica $\mathrm{CH}_{18}$ & KU176127 & & & & 27.49 & 19.43 & 28.24 & 24.84 & & 942 \\
\hline H. indica & JQ178379 & & & & 25.85 & 19.39 & 29.13 & 25.63 & & 913 \\
\hline H. amazonens & EU099036 & & & & 26.07 & 19.55 & 29.06 & 25.32 & & 936 \\
\hline H. floridens & EU099034 & & & & 25.56 & 19.79 & 29.3 & 25.35 & & 935 \\
\hline H. Mexicana & EU100414 & & & & 25.48 & 19.81 & 29.44 & 25.27 & & 934 \\
\hline H. bacteriophora & JQ178377 & & & & 25.49 & 19.89 & 29.45 & 25.16 & & 910 \\
\hline \multicolumn{11}{|l|}{ COXI } \\
\hline H. indica $\mathrm{CH}_{18}$ & KU306237 & & & & 23.83 & 12.75 & 19.3 & 44.13 & & 596 \\
\hline H. indica & AB355853 & & & & 22.81 & 9.81 & 19.89 & 47.48 & & 377 \\
\hline
\end{tabular}




\begin{tabular}{|c|c|c|c|c|c|c|}
\hline H. taysearae & EF043421 & 24.42 & 11.06 & 19.47 & 45.05 & 606 \\
\hline H. Mexicana & EF043422 & 24.92 & 10.4 & 18.81 & 45.87 & 606 \\
\hline H. bacteriophora & JQ423214 & 23.77 & 8.62 & 18.76 & 48.85 & 917 \\
\hline
\end{tabular}

The sequence of the D2-D3 region was $942 \mathrm{bp}$ long with nucleotide composition as: $A=27.49 \%, C=19.43$ $\%, G=28.24 \%, T=24.84 \%$. The sequence of the COX1 mtDNA region was 596 bp with nucleotide configuration: $A=23.83 \%, C=12.75 \%, G=19.30 \%, T=$ 44.13\% (Table 3).

For ITS regions, maximum parsimony analysis showed that the alignment resulted in 1019 characters, of which 267 were constant, 467 variable characters were parsimony uninformative and 285 characters were parsimony informative. Parsimony and distance based tree building approaches produced almost identical trees. The phylogenetic relationships between 16 Heterorhabditis species are presented in Fig. 1, with MP Tree length $=710$, Consistency Index = 0.74498 , Retention Index $=0.875$, Composite Index $=$ 0.718486 (0.651857). In this consensus tree, two species, $H$. indica (AY321483) and H. indica isolate $\mathrm{CH}_{18}$ form a monophyletic group with bootstrap support of $98 \%$. All positions containing gaps and missing data were eliminated. There were a total of 574 positions in the final dataset.

For D2-D3 region, maximum parsimony analysis showed the alignment resulted in 1034 characters, of which 174 are constant, 530 variable characters are parsimony uninformative and 330 characters are parsimony informative. The phylogenetic relationships between 17 Heterorhabditis species are presented in Fig. 2, with MP Tree length $=687$, Consistency Index = 0.814126, Retention Index $=0.862637$, Composite Index 0.799854 (0.702296). There were a total of 574 positions in the final dataset. In the consensus tree, $H$. indica and the present specimen along with two undescribed species form a monophyletic group.

For the COX1 gene, maximum parsimony analysis showed that the alignment resulted in 1034 characters, of which 105 are constant, 609 variable characters are parsimony-uninformative and 217 characters are parsimony-informative. The phylogenetic relationships between 17 Heterorhabditis species are presented in Fig. 3, with MP Tree length $=555$, Consistency Index $=0.679878$,
Retention Index $=0.562500$, Composite Index = 0.456081 (0.382431). There were a total of 354 positions in the final dataset. The present species showed deviation from the above results which might be due to non-utility of $\mathrm{CO} 1$ gene for identification purpose of EPN. 
Table 4. Pairwise distances of the ITS regions between $H$.indica $\mathrm{CH}_{18}$ and closely related species of Heterorhabditis. Below diagonal: \% age similarity; above the diagonal: no. of base substitutions per site between sequences, according to the Jukes-Cantor model. Data for isolate $\mathrm{CH}_{18}$ in bold.

\begin{tabular}{|c|c|c|c|c|c|c|c|c|c|c|c|c|c|c|c|}
\hline ITS region & $\mathrm{CH}_{18}$ & ind & $b a c$ & bau & noe & $a m a$ & flo & mex & geo & ata & saf & mar & zea & dow & meg \\
\hline $\mathrm{CH} 18$ & & 0.003 & 0.031 & 0.023 & 0.008 & 0.021 & 0.024 & 0.024 & 0.031 & 0.033 & 0.034 & 0.036 & 0.046 & 0.039 & 0.040 \\
\hline H. bacteriophora & 77 & 77 & & 0.034 & 0.032 & 0.033 & 0.035 & 0.035 & 0.006 & 0.024 & 0.024 & 0.026 & 0.035 & 0.027 & 0.028 \\
\hline H. baujardi & 86 & 86 & 74 & & 0.024 & 0.008 & 0.009 & 0.011 & 0.034 & 0.035 & 0.036 & 0.037 & 0.044 & 0.039 & 0.038 \\
\hline H. noenieputensis & 98 & 98 & 76 & 86 & & 0.022 & 0.024 & 0.025 & 0.031 & 0.033 & 0.034 & 0.036 & 0.045 & 0.039 & 0.040 \\
\hline H. amazonensis & 88 & 87 & 75 & 98 & 87 & & 0.010 & 0.012 & 0.033 & 0.035 & 0.036 & 0.037 & 0.044 & 0.040 & 0.039 \\
\hline H. floridensis & 85 & 85 & 73 & 97 & 85 & 97 & & 0.008 & 0.035 & 0.037 & 0.038 & 0.039 & 0.047 & 0.043 & 0.042 \\
\hline H. Mexicana & 85 & 84 & 73 & 96 & 84 & 96 & 98 & & 0.035 & 0.038 & 0.039 & 0.040 & 0.048 & 0.044 & 0.043 \\
\hline H. Georgiana & 78 & 77 & 99 & 74 & 77 & 75 & 73 & 98 & & 0.025 & 0.025 & 0.027 & 0.035 & 0.028 & 0.029 \\
\hline H. atacamensis & 74 & 73 & 85 & 71 & 74 & 72 & 69 & 98 & 85 & & 0.007 & 0.010 & 0.029 & 0.016 & 0.018 \\
\hline H. safricana & 73 & 72 & 86 & 71 & 73 & 71 & 68 & 97 & 85 & 98 & & 0.010 & 0.029 & 0.016 & 0.018 \\
\hline H. marelatus & 70 & 70 & 84 & 69 & 71 & 70 & 66 & 99 & 83 & 96 & 97 & & 0.029 & 0.017 & 0.019 \\
\hline H. zealandica & 61 & 60 & 73 & 62 & 61 & 62 & 58 & 98 & 73 & 79 & 79 & 79 & & 0.030 & 0.030 \\
\hline H. downesi & 68 & 68 & 82 & 68 & 68 & 68 & 64 & 99 & 81 & 93 & 93 & 91 & 79 & & 0.014 \\
\hline H. megidis & 66 & 65 & 79 & 68 & 66 & 67 & 64 & 99 & 78 & 90 & 90 & 89 & 78 & 94 & \\
\hline
\end{tabular}


Table 5. Pairwise distances of the D2D3 regions between Heterorhabditis indica $\mathrm{CH}_{18}$ and closely related species of Heterorhabditis. Below diagonal: \%age similarity; above the diagonal: no. of base substitutions per site between sequences, according to the Jukes-Cantor model. Data for isolate $\mathrm{CH}_{18}$ in bold.

\begin{tabular}{|c|c|c|c|c|c|c|c|c|c|c|c|c|c|}
\hline D2D3 egion & $\mathrm{CH}_{18}$ & ind & $a m a$ & mex & noe & flo & saf & mar & $b a c$ & ata & geo & zea & meg \\
\hline $\mathrm{CH} 18$ & & 0.002 & 0.008 & 0.008 & 0.002 & 0.008 & 0.011 & 0.012 & 0.011 & 0.011 & 0.011 & 0.297 & 0.012 \\
\hline H. indica & 100 & & 0.008 & 0.008 & 0.002 & 0.008 & 0.011 & 0.012 & 0.010 & 0.011 & 0.011 & 0.296 & 0.012 \\
\hline H. amazonensis & 96 & 96 & & 0.003 & 0.008 & 0.003 & 0.011 & 0.011 & 0.010 & 0.011 & 0.010 & 0.288 & 0.012 \\
\hline H. Mexicana & 96 & 96 & 99 & & 0.008 & 0.003 & 0.012 & 0.012 & 0.010 & 0.011 & 0.011 & 0.287 & 0.012 \\
\hline H. noenieputensis & 100 & 100 & 96 & 96 & & 0.008 & 0.011 & 0.012 & 0.010 & 0.011 & 0.011 & 0.295 & 0.012 \\
\hline H. floridensis & 96 & 96 & 99 & 99 & 96 & & 0.012 & 0.012 & 0.010 & 0.011 & 0.010 & 0.287 & 0.012 \\
\hline H. safricana & 94 & 94 & 93 & 93 & 94 & 93 & & 0.004 & 0.009 & 0.004 & 0.009 & 0.272 & 0.006 \\
\hline H. marelatus & 93 & 93 & 93 & 93 & 93 & 93 & 99 & & 0.009 & 0.004 & 0.009 & 0.273 & 0.006 \\
\hline H. bacteriophora & 93 & 94 & 94 & 94 & 94 & 94 & 95 & 95 & & 0.008 & 0.003 & 0.303 & 0.010 \\
\hline H. atacamensis & 93 & 93 & 94 & 94 & 93 & 93 & 99 & 99 & 96 & & 0.008 & 0.272 & 0.006 \\
\hline H. Georgiana & 93 & 93 & 94 & 94 & 93 & 94 & 95 & 95 & 99 & 96 & & 0.305 & 0.010 \\
\hline H. zealandica & 49 & 50 & 48 & 48 & 48 & 50 & 41 & 42 & 53 & 42 & 55 & & 0.273 \\
\hline H. megidis & 92 & 92 & 93 & 25 & 92 & 92 & 98 & 98 & 94 & 98 & 94 & 42 & \\
\hline
\end{tabular}




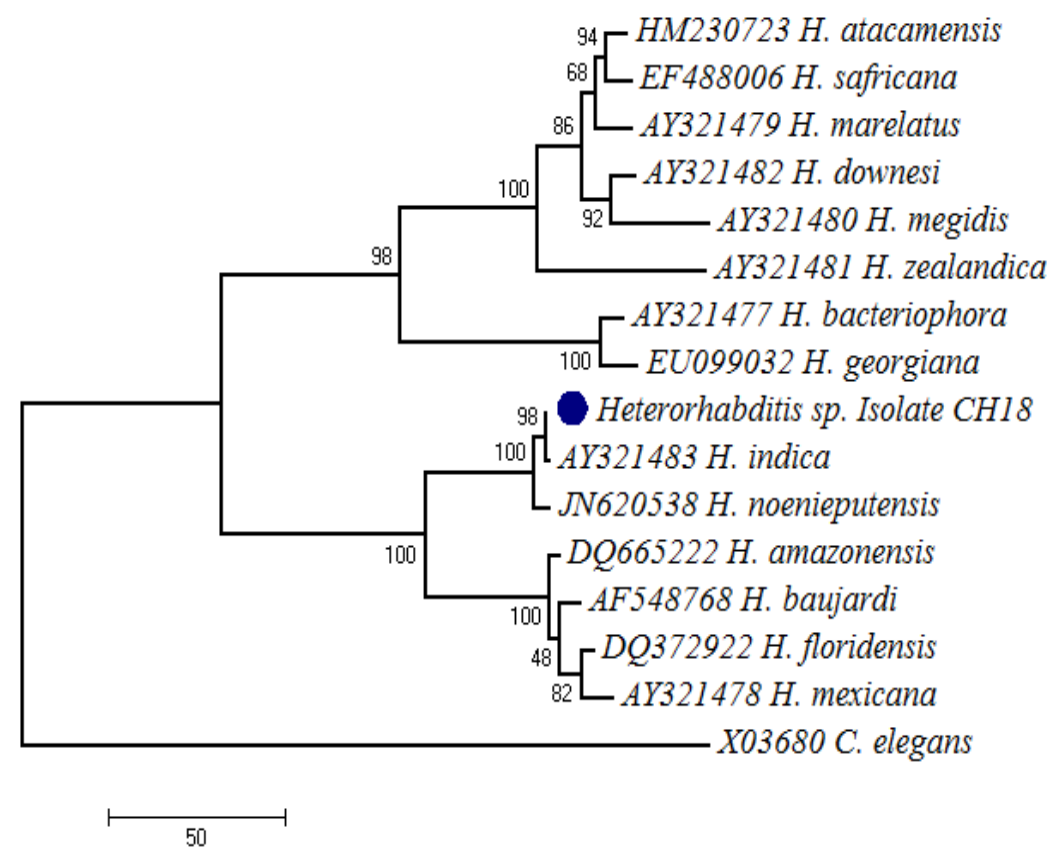

Fig. 1. Phylogenetic analyses of ITS region of isolate $\mathrm{CH}_{18}$ and other species of Heterorhabditis. The dendrogram was constructed by Maximum Parsimony method. Bootstrap values are included.

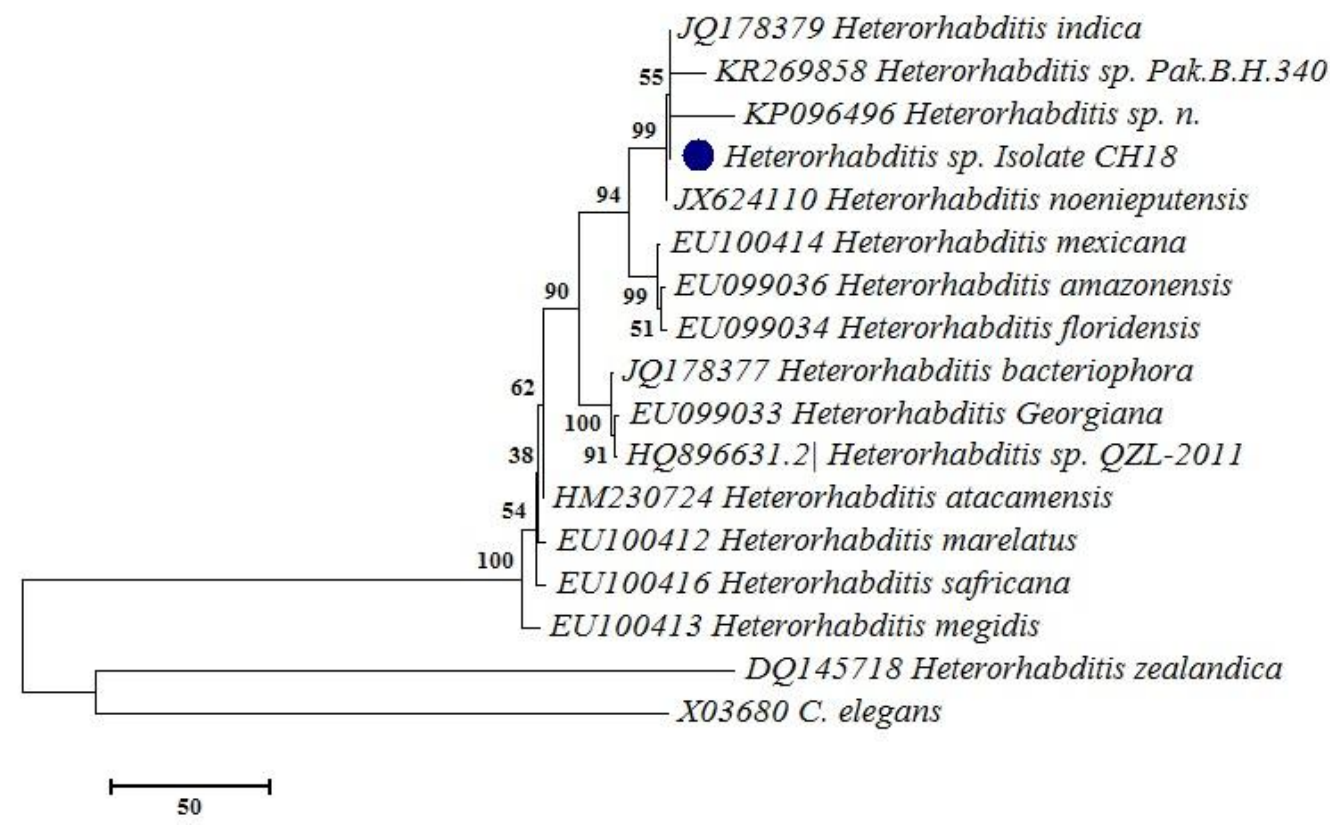

Fig. 2. Phylogenetic relationships based on Maximum Parsimony between 17 Heterorhabditis species and isolate $\mathrm{CH}_{18}$ with bootstrap analysis of D2D3 regions. 


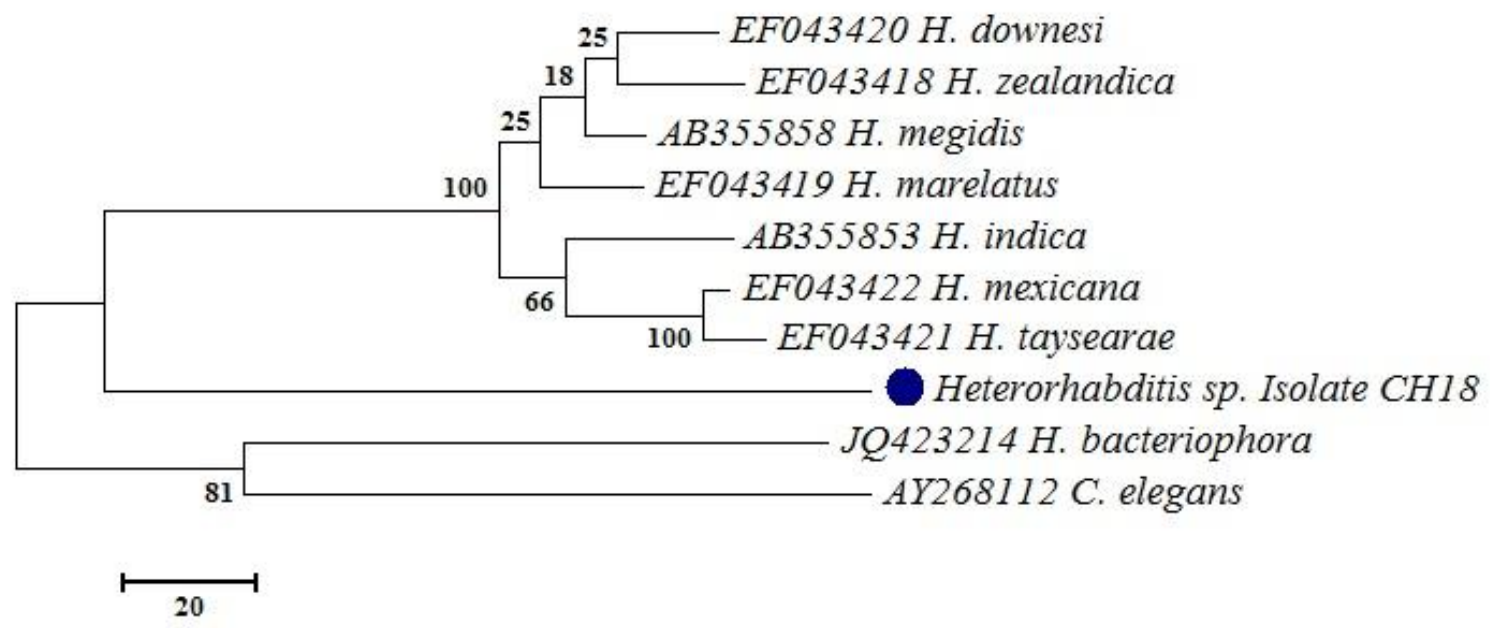

Fig. 3. Phylogenetic relationships based on Maximum Parsimony between 10 Heterorhabditis species and isolate $\mathrm{CH}_{18}$ with bootstrap analysis of $\mathrm{COI}$ regions.

\section{Pathogenicity}

The evaluation of the pathogenicity and reproductive potential of $\mathrm{H}$. indica isolate $\mathrm{CH}_{18}$ was carried out in laboratory condition against $G$. mellonella as target host using different IJ concentration viz. 25, 50, 100, $200 \mathrm{IJ} /$ larva. At $12 \mathrm{~h}$ post infection period, no mortality was recorded in all infected larva against the applied doses, while 10, 30, 30 and 90\% mortality was observed in 25, 50, 100, $200 \mathrm{JJ} /$ larva, respectively after $24 \mathrm{~h}$ post infection period (Fig. 4). At $36 \mathrm{~h}$ post infection period $100 \%$ mortality was noticed in 200 $\mathrm{IJ} /$ larva dose followed by 70,60 , and $40 \%$ respectively in 100, 50 and $25 \mathrm{JJ} /$ larva. At $48 \mathrm{~h}$ post infection period, $90 \%$ mortality was observed in $100 \mathrm{IJ} /$ larva while $80 \%$ was shown in 25 and $50 \mathrm{IJ} /$ larva. At $60 \mathrm{~h}$ post infection period, $100 \%$ was discerned in all the concentrations. However, the degree of susceptibility to nematode infection varied from different dose infection and also on the exposure time. No mortality was recorded in control group even after $60 \mathrm{~h}$ during the experiment. All the dead larvae were transferred on the modified white trap to confirm their death was due to EPN where the emergence of IJ from the cadaver was the sign of nematode infection. A positive relation was observed in between the doses of infective juvenile and larval mortality where increments in doses also lead to increment in mortality rate.

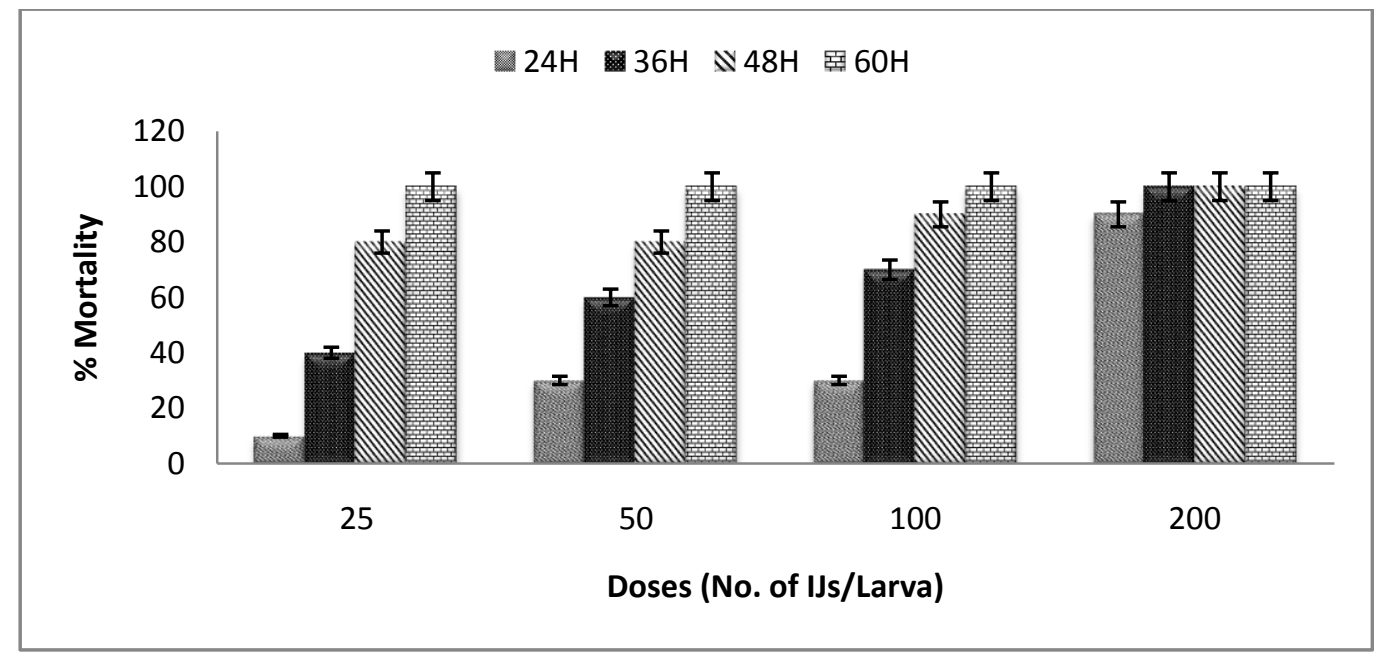

Fig. 4. Percentage mortality of G. mellonella larvae treated with $H$. indica isolate $\mathrm{CH}_{18}$. 


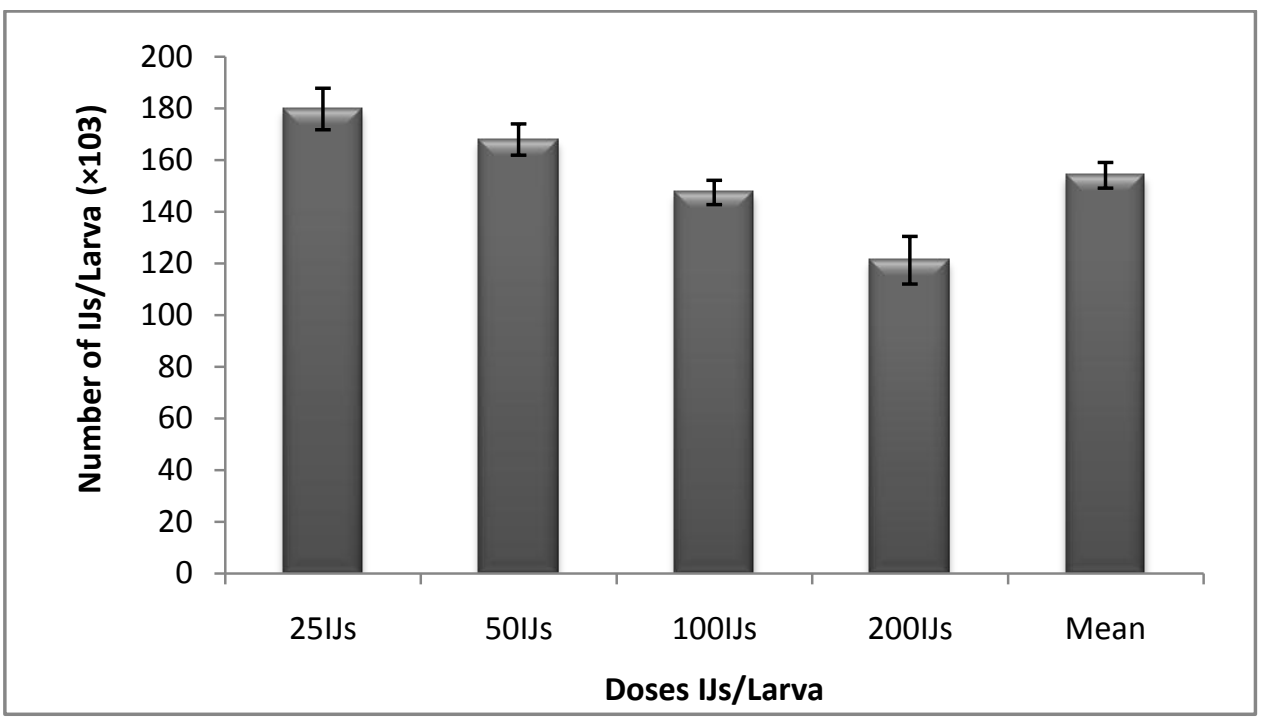

Fig. 5. Progeny productions $\mathrm{H}$. indica isolate $\mathrm{CH}_{18}$ in $\mathrm{G}$. mellonella larvae at different doses of IJ.

$\mathrm{LC}_{50}$ and $\mathrm{LC}_{90}$ value were also calculated to judge the pathogenicity of isolate by probit analysis at three different post infection periods viz. 24, 36 and $48 \mathrm{~h}$ and presented in IJ/Larva. Higher $\mathrm{LC}_{50}$ value was recorded at $24 \mathrm{~h}$ post infection period which was about $96 \mathrm{IJ} /$ Larva followed by $37 \mathrm{IJ} /$ Larva at $36 \mathrm{~h}$ post infection period where as lowest $\mathrm{LC}_{50}$ value was reported after $48 \mathrm{~h}$ which was only $8 \mathrm{IJ} /$ Larva confirming the high pathogenicity of present isolate. $\mathrm{LT}_{50}$ and $\mathrm{LT}_{90}$ values were also figured out at different concentrations viz. 25, 50, 100 and $200 \mathrm{IJ} /$ Larva and for mean doses too through same analysis. The lowest time $\left(L T_{50}\right)$ was taken with high dose which was only $19.3 \mathrm{~h}$ followed by $29.5,31.4$ and $36.8 \mathrm{~h}$ with 100,50 and $25 \mathrm{IJ} /$ Larva respectively. The mean $\mathrm{LT}_{50}$ calculated for all the doses applied was 25.7 hours.

The above findings showed similitude with that of the Sankar et al. ${ }^{20}$ and with that of Hara and Kaya ${ }^{21}$ which reported that nematodes are symbiotically associated with mutualistic bacteria, which allow them to kill their hosts quickly ( 36 to $48 \mathrm{~h}$ ) and thus give them an advantage over other parasitoids and pathogens. Divya et al. ${ }^{22}$ revealed highest death rate of the greater wax moth larvae at concentration levels of $300 \mathrm{IJ} /$ larva within $24 \mathrm{~h}$ exposure of $\mathrm{H}$. indica but the present Heterorhabditis isolate showed $100 \%$ mortality with different concentrations viz., 25, 50, 100 and 200 only after $36 \mathrm{~h}$ of exposure. This may be due to the fact the pathogenicity being complex process, depends upon many biotic and abiotic factors as was reported by Kaya and Gaugler ${ }^{23}$.

\section{Reproductive potential}

In vivo progeny production of infective juveniles was also performed to assess the reproductive potential at different concentrations for same larvae used in pathogenicity bioassay. The IJ production was recorded as number of IJ/Larva. Descriptive analysis for IJ production revealed that the population count of IJ decreased with increase in increment of dose. The highest progeny production was recorded $1.79 \times 105$ (140800-217600) at $25 \mathrm{IJ} /$ Larva infection whereas lowest was recorded at 200 IJ/Larva 1.21×105 (86400-172800). At 50 and $100 \mathrm{IJ} /$ Larva, progeny count calculated were $1.68 \times 10^{5}$ (147200$211200)$ and $1.47 \times 10^{5}(121600-169600)$, respectively (Fig. 4).

The overall effectiveness of pest control program is directly related to the reproductive capability of EPN within the host and determines the time and dose applied in the field ${ }^{24,25}$. In conjugation with this, it is far too expensive to rear EPN by in vitro media as they required a lot of care, instruments and subsequent labor practices. Hence the species/strain of high yielding progeny is a requisite of the biological control program. Reproductive capability of different nematode species to produce infective juveniles was different $^{26,27}$. Apart from this, the size of the nematode and their behaviour directly influenced the reproduction of nematode species within the body of 
host $^{28}$ as reported by Bhatnagar et al. ${ }^{29}$ in $H$. bacteriophora. Availability of food also influenced the number of eggs in the uterus of hermaphrodite by delaying the endotokia matricida ${ }^{30}$.

Analysis of variance (ANOVA) indicated that there was negative correlation in between the production of IJ and doses applied where the significance value was zero. The positive relationship between the dose of IJ and host mortality found in the present examination has also been documented in several other investigations ${ }^{31,32,33}$. Gupta et al. ${ }^{34}$ reported gradual increase in progeny production up to $160 \mathrm{IJ}$ of $S$. carpocapsae per larva of Pieris brassicae, after which a sudden decrease from $2.20 \times 105$ larva to $1.70 \times 105$ was noticed. Small size of $\mathrm{H}$. indica isolate $\mathrm{CH}_{18}$ and hermaphroditism could be the reason of the high yield of progeny as compared to others and supported by several findings ${ }^{7,35,36}$. However, in the present study, the highest reproduction was in low dose $(25 \mathrm{lJ} /$ Larva dose with 1.79 lacs IJ/Larva) as compared to other researchers with different/ same species in different/ same insect host.

\section{ACKNOWLEDGEMENTS}

Authors are grateful to the Department of Science and Technology (DST), New Delhi, India for providing the financial assistance through DST INSPIRE Fellowship/2014/76 and University Grants Commission (UGC), New Delhi, India for providing support via Rajeev Gandhi National Fellowship (F117.1/2015-16/RGNF-2015-17-SC-UTT-183610).

\section{REFERENCES}

1. Kaya HK., in: Hoy MA, Herzog DC, (Eds.), Biological control in agricultural IPM systems, New York, Academic Press, 1985, pp. 283-302.

2. Gaugler R. Ecological considerations in the biological control of soil inhabiting insects with entomopathogenic nematodes. Agric Ecosyst and Environ, 24: 351-360, (1988)

3. Gaugler R., Kaya HK, Entomopathogenic nematodes in biological control, Boca Raton, FL, USA, CRC Press 1990, 365.

4. Boemare NE, Akhurst RJ, Mourant RG. DNA relatedness between Xenorhabdus spp. (Enterobacteriaceae), symbiotic bacteria of entomopathogenic nematodes, and a proposal to transfer Xenorhabdus luminescens to a new genus, Photorhabdus gen. nov. Int J Syst Bacteriol, 43: 249-255, (1993).

5. Thomas GM, Poinar GO Jr. Xenorhabdus gen. nov. a genus of entomopathogenic and nematophilic bacteria of the family Enterobacteriaceae, Int J Syst Bacteriol, 29: 352-360, (1979).

6. Curran J, Webster JM. Genorypic analysis of Heterorhabditis isolates from North Carolina. J Nematol, 21: 140-145, (1989).

7. Poinar GO Jr., in: Gaugler R., and Kaya HK., (Eds.). Taxonomy and biology of Steinernematidae and Heterorhabditidae. Entomopathogenic nematodes in biological control, CRC Press, Boca Raton, 23-61, (1990).

8. Akhurst R, Bedding RA. A simple crossbreeding technique to facilitate species determination in the genus Neoaplectana, Nematol, 24: 328-330, (1978).

9. Poinar, GO Jr., in: Frenz, JM (Eds.), Entomopathogenic nematodes in biological plant and health protection. Entomogenous nematodes, Stuttgart, Germany, G. Fischer Verlag, 32 : 95-121 (1986).

10. Curran J., in: Gaugler R and Kaya HK. (Eds.), Molecular techniques in taxonomy, Entomopathogenic nematodes in biological control, Boca Raton FL USA CRC Press, 63-74 (1990)

11. Curran J, Driver F, Ballard JWO, Milner RJ. Phylogeny of Metarhizium: analysis of ribosomal DNA sequence data. Mycol Res, 98: 547-552, (1994).

12. Reid, AP., in: Burnell, AM, Ehlers, RU, Masson, JP, (Eds.), Molecular taxonomy of Steinernema, COST 812 Biotechnology: Genetics of entomopathogenic nematodes-bacterium complexes, ProcSymp Works St. Patrick's College, Maynooth, County Kildare Ireland Luxembourg DG XII, European Commission, (1994).

13. Stock SP, Goodrich-Blair H. Nematode parasites, pathogens and associates of insects and invertebrates of economic importance. Lacey LA 90 Manual of techniques in invertebrate pathology, $2^{\text {nd }} E d n$, Amsterdam, The Netherlands, Elsevier, 373-426, (2012).

14. Woodring, JL, Kaya, HK., in: Steinernematid and Heterorhabditid nematodes: A Handbook of Biology and Techniques, South Cooperative Services Bulletin 331. Arkansas Agricultural Experiment Station Fayetteville, Arkansas, (1988).

15. White GF. A method for obtaining infective nematode larvae from cultures, Science, 66: 302-303, (1927)

16. Courtney WD, Polley D, Miller VI. TAF, an improved fixative in nematode technique, Plant Dis Repr, 39: 570-571, (1955) 
17. Seinhorst JW. A rapid method for the transfer of nematodes from fixative to anhydrous glycerine, Nematol, 4: 117-128, (1959)

18. Joyce SA, Reid AP, Driver F, Curran J. Application of polymerase chain reaction (PCR) methods to identification of entomopathogenic nematodes, Proc Symp Work, St Patrick's College, Maynooth, Co Kildare, Ireland. Luxembourg, European Commission, DGXII, 178-187 (1994).

19. Tamura K, Stecher G, Peterson D, Filipski A, Kumar S. MEGA 6: Molecular Evolutionary Genetics Analysis version 6.0. Mol Biol Evol, 30: 2725-2729, (2013)

20. Sankar M, Sethuraman V, Palaniyandi M, Prasad JS. Entomopathogenic nematode- Heterorhabditis indica and its compatibility with other biopesticides on the Greater wax moth, Galleria mellonella (L), Ind J Sci Technol, 2: 57-62, (2009)

21. Hara AH, Kaya K. Effect of selected insecticides and nematicides on the in vitro development of the entomogenous nematode Neoplectana carpocapsae, J Nematol, 14: 486-491, (1982)

22. Divya K, Sankar M, Marulasiddesha KN. Efficacy of Entomopathogenic nematode, Heterorhabditis indica against three Lepidopteran insect pests, Asian J Biol Sci, 1: 183-188, (2010)

23. Kaya HK, Gauglar R. Entomopathogenic nematodes, Ann Rev Entomol, 38: 181-206, ( 1993)

24. Harlan DP, Dutky SR, Padgett GR. Mitchell JA. Shaw ZA, Barlett FJ. Parasitism of Neoaplectana dutkyi in whitefringed beetle larvae, J Nematol, 3: 280-283, (1971)

25. Georgis R, Hague NGM. A neoaplectanid nematode in the larch sawfly Cephalcia lariciphila (Hymenoptera: Pamphiliidae), Ann Appl Biol, 99: 171-177, (1981)

26. Karunakar G, Easwaramoorthy S, David H. Susceptibility of nine lepidopteran insects to Steinernema glaseri, S. feltiae and Heterorhabditis indicus infection. Int J Nematol, 9: 68-71, (1999)

27. Ali SS, Perveza R, Hussain MA, Ahmad R. Susceptibility of three lepidopteran pests to five entomopathogenic nematodes and in vivo mass production of these nematodes, Arch Phytopathol, 41: 300-304, (2006)

28. Loya LJ, Hower AA. Infectivity and reproductive potential of the Oswego strain of Heterorhabditis bacteriophora associated with life stages of the clover root curculio, Sitonahispidulus, J Invertebr Pathol, 83: 63-72, (2003)

29. Bhatnagar A, Shinde V, Bareth S S. Evaluation of entomopathogenic nematodes against white grub, Maladerainsanabilis Brenske, Int J Pest Manage, 50: 285-289, (2004)

30. Johnigk SA, Ehlers RU. Endotokiamatricida in hermaphrodites of Heterorhabditis spp. and the effect of the food supply, Nematol, 1: 717-726, (1999)

31. Forschler BT, Nordin GL. Comparative pathogenicity of selected entomogenous nematodes to the hardwood borers, Prionoxystus roblniae (Lepidoptera: Cossidae) and Megacylletzev obiniae (Coleoptera: Cerambycidae), J InvertebrPathol, 52: 343-347, (1988)

32. Glazer L, Navon A. Activity and persistence of entomoparastitic nematodes tested against Heliothisarmigera, J Eco Entomol, 83: 1795 -1800, (1990)

33. Peters A, Ehlers RU. Susceptibility of leather jackets (Tipulapaludosa and Tipulaoleracea; Tipuladae: Nematocera) to the entomopathogenic nematode Steirnema feltiae, J Invertbr Pathol, 63: 163-171, (1994)

34. Gupta S, Kaul V, Shankar U. Progeny production and pathogenicity of local isolate of Steinernema carpocapsae against Pieris brassicae (L.), Green Farming, 1: 27-30, (2008)

35. Mannion CM, Jansson RK. Comparison of ten entomopathogenic nematodes for control of sweet potato weevil (Coleoptera: Apionidae), J Econ Entomol, 85: 1642-1650, (1992)

36. Jothi DB, Mehta UK. Pathogenicity of three species of EPN against cotton bollworm Helicoverpa armigera Hub, Entomon, 31: 259-266, (2006)
*Corresponding Author: Aashaq Hussain Bhat ${ }^{*}$ Email: aashiqhussainbhat10@gmail.com 